J. Clin. Chem. Clin. Biochem.

Vol. 14, 1976, pp. 205-211

\title{
Eine schnelle und exakte Fibrinogen-Bestimmungsmethode unter Berücksichtigung von Spaltprodukten
}

\author{
Von $H$. Ebeling \\ Institut für Klinische Chemie und Klinische Biochemie (Direktor: Prof. Dr. H.-J. Dulce) am Klinikum Steglitz der \\ Freien Universität Berlin
}

(Eingegangen am 1. Dezember 1975/5. Februar 1976)

Zusammenfassung: Mit einer mechanisierten nephelometrischen Meßtechnik werden immunologisch-faßbare Fibrinogenäquivalente vor und nach Ausgerinnen des Fibrinogens aus Citratblut quantitativ bestimmt. Man erhält so einerseits die Summe aller Fibrinogenäquivalent-Konzentrationen und andererseits die „Spaltprodukt"-Konzentrationen. Durch Differenzbildung aus beiden Messungen ergibt sich die Konzentration an gerinnbarem Fibrinogen.

Für eine Fibrinogen-Konzentration von $11,15 \mathrm{mg} / \mathrm{l}$ ergeben sich Variationskoeffizienten von $2,81 \%$ pro Serie und 3,31\% von Tag zu Tag.

Im Vergleich mit der einfachen radialen Immundiffusion und der ,gerinnungsphysiologischen Schnellmethode zur Bestimmung des Fibrinogens" nach Clauss ((1957), Acta Haematol. 17, 237-246) wird unter Fibrinogenolyse-Bedingungen und unter Heparin die „Richtigkeit“ der mit der neuen Methode erhaltenen Werte überprüft.

Zusätzlich wurden aus 180 Citratblutproben eines gemischten Krankenguts im Methodenvergleich mit der einfachen radialen Immundiffusion und der Methode nach Clauss Fibrinogen-Bestimmungen durchgeführt.

Nur die neue Schnellmethode mißt offensichtlich plausible Fibrinogenwerte und läßt gut zwischen gerinnbaren und nicht gerinnbaren Fibrinogenäquivalenten unterscheiden. Sie empfiehlt sich für die Klinik.

\section{$A$ rapid and precise determination of fibrinogen and its cleavage products}

Summary: A method is described for the quantitative determination of fibrinogen equivalents in citrated blood before and after removal of the fibrinogen by coagulation. The method employs an immunological reaction, which is measured by mechanized nephelometry. Thus the sum of all the fibrinogen equivalents can be compared with the concentration of "cleavage products". The difference between the two readings represents the concentration of coagulable fibrinogen. For a fibrinogen concentration of $11.15 \mathrm{mg} / \mathrm{l}$, variation coefficients of $2.81 \%$ in series and $3.31 \%$ for day to day were obtained.

The accuracy of the new method was tested under conditions of fibrinogenolysis, and in the presence of heparin, by comparison with simple radial immunodiffusion, and with the "coagulation physiological rapid method for the determination of fibrinogen" after Clauss ((1957), Acta Haematol. 17, 237-246).

In addition 180 citrated blood samples of different patients were compared with the simple radial immunodiffusion, the method of Clauss and the new method.

Only the new rapid method appears to measure plausible fibrinogen values, and permits a clear differentiation between coagulable and non-coagulable fibrinogen equivalents. It can be recommended for clinical practice.

\section{Einleitung ǜ Fragestellung}

Für die Klinik sind die Zustände der Verbrauchskoagulopathie und Fibrinogenolyse bei haemorrhagischen Diathesen mèhr und mehr in deri Mittelpunkt des Interesses gerückt. Dies bedingt, daß besonders in kritischen Situationen nur verläßliche und empfindliche Fibrinogen-
Bestimmungsmethoden als Labortests verwendet werden dürften.

Anliegen dieser Arbeit war es, eine Methode zu entwickeln, die schnell, empfindlich und exakt Fibrinogenwerte mißt, auch und gerade unter extremen Krankheitsund Therapie-Bedingungen wie Fibrinogenolyse und hoher Heparin-Dosierung. 


\section{Material und Methoden}

\section{Apparate}

Technicon Auto Analyzer, wie beschrieben $(2,3)$, hier zweikanalig. Fließschema s. Abbildung 1.

Coagulometer nach Schnitger \& Groß (Fa. H. Amelung, LemgoBrake).

\section{Reagenzien}

Standard-Human-Plasma,

Human-Plasmaprotein-Anti-Fibrinogen-Serum vom Kaninchen, Test-Thrombin

Test-Streptokinase (5000 I.E./2,5 ml) und Immundiffusionsplatten M-Partigen-Fibrinogen (Fa. Behringwerke AG, Marburg).

Natriumcitratlösung 3,8\% (Fa. Dr. E. Fresenius KG, Bad Homburg v. d. H.).

Trasylol $100000 \mathrm{KIE} / 5 \mathrm{ml}$ (Fa. Bayer AG, Leverkusen). Liquemin 20000 USP-E. Heparin-Natrium/ml (Fa. Hoffmann-La Roche AG, Grenzach, Baden).

Owrens-Veronal-Puffer pH 7,35, 175,5 mmol/1:

$\mathrm{NaCl} \quad 125,7 \mathrm{mmol} / \mathrm{l}$

Barbital-Natrium p.a. $28,3 \mathrm{mmol} / 1$ und

$\mathrm{HCl}$

21,5 mmol/1 (Fa. E. Merck, Darmstadt).

Britton-Robinson-Puffer-NaCl-Lösung pH 6,00, $11 \mathrm{mmol} / \mathrm{l}(2,4)$. Trasylol-Lösungen: Verdünnungen mit Britton-Robinson-PufferNaCl-Lösung 1:4 für Methode nach Clauss (1) und Partigenplatten-Methode (zitiert nach 1.c. (5)), für das standardisierte -nephelometrische Vorgehen 1:200.

Test-Thrombin-Lösung I für Methode nach Clauss: nach der Vorschrift des Herstellers gelöst ( $=10 \mathrm{NIH}-\mathrm{E} \cdot / 0,1 \mathrm{ml})$.
Test-Thrombin-Lösung II für nephelometrische Methode: gelöst in Britton-Robinson-Puffer-NaCl-Lösung ( $\triangleq 2,5 \mathrm{NIH}-\mathrm{E} . / 50 \mu \mathrm{l})$. Test-Streptokinase-Lösung: 5000 I.E./2,5 ml Britton-RobinsonPuffer-NaCl-Lösung.

Heparin-Lösungen: Liquemin-Verdünnungen mit Britton-Robinson-Puffer-NaCl-Lösung.

Reagenzien zur zweidimensionalen Geldoppeldiffusion, wie beschrieben (2).

\section{Probenmaterial}

Citratblut ( $=1$ Teil Natriumcitratlösung +9 Teile Blut) wird mit einer Plastikspritze entnommen und für 10 Minuten bei $1500 \mathrm{~g}$ in silikonisierten Glasröhrchen zur Citratplasma-Gewinnung zentrifugiert.

Für die Methode nach Clauss und die einfache radiale Immundiffusion auf der Partigenplatte diente als gemeinsames Ausgangsmateriäl eịne 1:2-Citratplasma-Vorverdünnung folgender Zusammensetzung:

$500 \mu$ l. Citratplasma

$50 \mu \mathrm{l}$ Trasylol-Lösung $(\triangleq 250 \mathrm{KIE})$

$450 \mu \mathrm{l}$ Owrens-Veronal-Puffer.

Für Fibrinogen-Konzentrationen außerhalb der Standardkurven wurden mit $O$ wrens-Veronal-Puffer andere Verdünnungen angesetzt.

Für die nephelometrische Arbeitsweise empfiehlt sich folgendes standardisiertes Vorgehen beim Ansetzen der Proben:

$$
10 \mu \text { l Citratplasmá }
$$

$100 \mu$ Trasy̆lol-Lösung ( $\triangleq 5 \mathrm{KIE})$

$2300 \mu$ Britton-Robinson-Puffer-NaCl-

Lösung. Diese 1:241-Citratplasma-Vorverdünnung ist das Ausgangsmaterial für die nephelometrische FibrinogenäquivalentBestimmung. Es werden zusammengegeben

a) zur Fibrinogenäquivalent-Bestimmung aus Citratplasma $1000 \mu 1$ 1:241-Citratplasma-Vorverdünnung

$50 \mu 1$ Britton-Robinson-Puffer $-\mathrm{NaCl}$ Lösung und

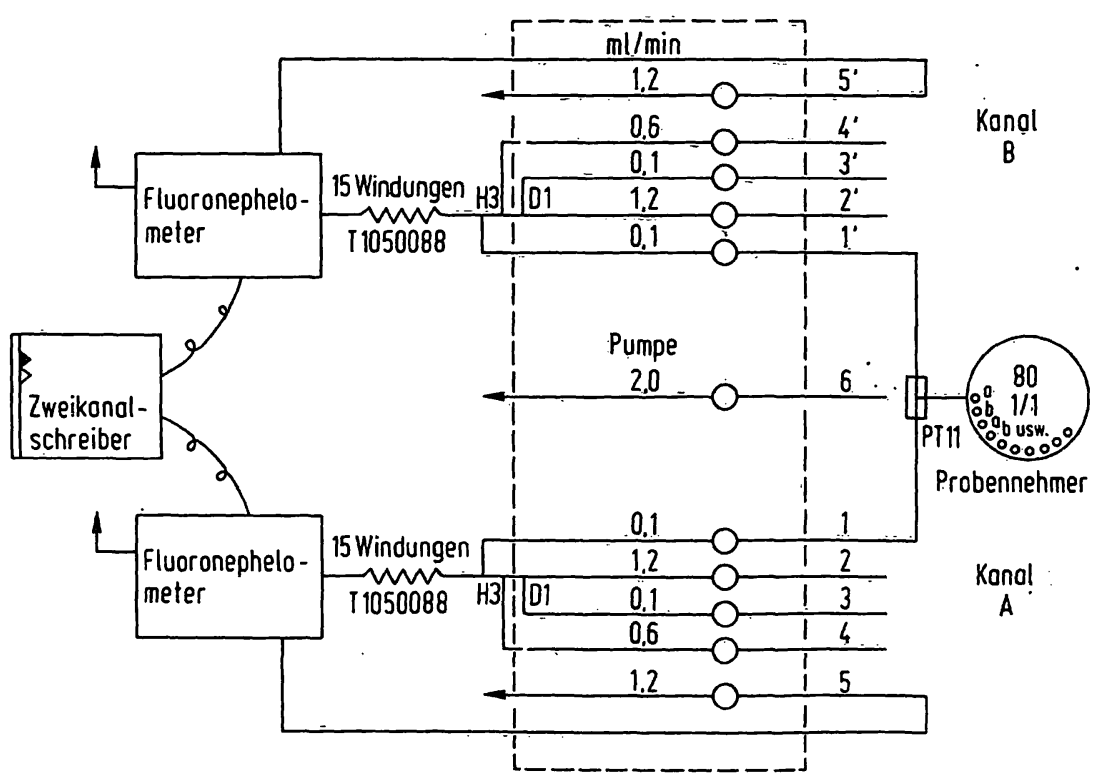

Abb. 1. Fließschema zum Vorgehen bei Fibrinogenäquivalent-Bestimmungen aus Plasma und Serum:

Kanal A: Antigen-Antikörper-Reaktion,

Kanal B: Proben-Eigenstreuung.

Dosier-Pumpenschläuche:

1 und $1^{\prime}=$ Antigenprobe (,,a“ = Plasma-, „,b" = „Serum"-Fibrinogenäquivalente),

2 und $2^{\prime}=$ Puffer-Lösung,

3 = Anti-Humanfibrinogen-Serum-Lösung,

$3^{\prime}$ = Puffer-Lösung,

4 und $4^{\prime}=$ Luft über Luftschleusen,

5 und $5^{\prime}=$ Küvettenabsaugung,

6 = Puffer-Lösung zum Probennehmer-Zwischenspülgefäß.

Vor den Bestimmungen ist auf exaktes nephelometrisches Abgleichen beider Fluoronephelometer streñg żu achten. 
b) zur Fibrinogenäquivalent-Bestimmung aus Citratserum $1000 \mu$ 1:241-Citratplasma-Vorverdünnung

$50 \mu l$ Test-Thrombin-Lösung II $(\triangle 2,5$ NIH-E.).

Nach mindestens 5 Minuten Reaktionszeit bei Raumtemperatur werden die Proben a) und b) in der Ecco-Zentrifuge bei $11000 \mathrm{~g}$ für 10 Minuten zentrifugiert. Die sich ergebenden Überstände des nun 1:253,05 vorverdünnten Citratplasmas werden als Proben a) und b) bei der mechanisierten, immunologisch-nephelometrischen Methode eingesetzt (siehe auch Fließschema, Abb. 1). Sofern eine sofortige Bestimmung der Proben nicht durchgefuhrt werden kann, empfiehlt sich Stapelverarbeitung, die hier besonders aus Vergleichsgründen mit den Methoden untereinander zweckmäßig war. Dazu wurden auf Abruf bis zu vier Wochen die beschriebenen 1:2-bzw. 1:241-Citratplasma-Vorverdünnungen bei $-20^{\circ} \mathrm{C}$ eingelagert.

Fibrinogenäquivalente-Bestimmung mit der neuen mechanisierten im munologisch-nephelometrischen Methode

Im Prinzip werden die Bestimmungen mit gegenüber früher beschriebenen leicht modifizierten Fließschemata durchgefuhrt $(2,3)$. Auch beim Zweikanal-Betrieb (siehe Abb. 1) wird sinngemäß so vorgegangen, wie bereits fir andere Systeme beschrieben $(2-4)$. Als standardisiertes optimales Reaktionsmilieu hat sich wiederum Britton-Robinson-Puffer-NaCl-Lösung mit pH 6,00 und einer Gesamtsalzkonzentration von $11 \mathrm{mmol} / 1$ bewährt. Als Antikörper diente auch hier Kaninchenserum, monospezifisch für Human-Plasma-Fibrinogen. In Abhängigkeit von den verwendeten Chargen benutzten wir 1:6- bis 1:12-Antiserum-Vorverdünnungen in Britton-Robinson-Puffer-NaCl-Lösung.

Ebenso wie für die einfache radiale Immundiffusion auf der Partigenplatte und die Methode nach Clauss wurde hier mit Standard-Human-Plasma die Fibrinogen-Kalibrierung vorgenommen. Für die Eichung empfehlen sich Fibrinogenäquivalent-Konzentrationen im Bereich von 40 bis $0,5 \mathrm{mg} / \mathrm{l}$. Dabei sollten besonders im unteren Konzentrations-Bereich ab 4,0 mg/l die Verdünnungsschritte kleiner sein, um vor allem die Serum-Fibrinogenäquivalente in diesem Konzentrationsbereich genau zu erfassen.

Erfahrungen wurden mit dem ein- wie dem zweikanaligen, mechanisierten Fibrinogen-Bestimmungs-System gesammelt. Da aber pro Patientenmaterial zwei Proben, a) und b), anfallen und korrekterweise beide Proben-Eigenstreuungen ebenfalls bestimmt werden müssen, erbringt ein zweikanaliges Vorgehen doppelten Zeitgewinn. So empfiehlt sich z. B. auf Kanal „A“ die AntigenAntikörper-Reaktion ablaufen zu lassen und über Kanal „B“ die dazugehörige Proben-Eigenstreuung zu bestimmen.

Bei einer Probennehmer-Frequenz von 80 pro Stunde, einem zeitlichen Probe-Ansaug- und Zwischen-Spüi-Verhältnis von 1:1 und einer reinen Reaktionszeit von 90 Sekunden erhält man beim Zweikañal-Betrieb in 320 Sekunden nach Ansaug-Beginn der ersten Probe sowohl die beiden „Antigen-Antikörper-plusEigenstreuungs"-Peaks als auch die dazugehörigen beiden „Eigenstreuungs"“Peaks auf dem Schreiberpapier registriert. Durch Peaklängen-Differenzzbildung zwischen den ,AntigenAntikörper-plus-Eigenstreuungs"-Peaks und dẹn, Eigenstreuungs":-Peaks ergeben sich

a) die ,reinen“ Antigen-Antikörper-Peaks der CitratplasmaFibrinogenäquivalent-Bestimmung und

b) die der Citratserum-Fibrinogenäquivalent-Bestimmung, hiẹ auch als ,Spaltprodukte" bezeichnet.

Anhand der mitgefahrenen Standardwerte werden die Konzen: trationen dèr ,reinen" Peak-Längen ermittelt. Multiplikation mit den Verdünnungsfáktoren ergeben die Fibrinogenäquivalent-Konzentrationen im Vollplasma und Vollserum. Subtraktion a) minus b) ergibt die gesuchte gerinnbare FibrinogenKonzentration im Vollplasma.

\section{Ergebnisse und Diskussion}

Aus Tabelle 1 sind die für diese Arbeit ermittelten methodenspeziifischen Qualitätskontröll-Angaben für die drei Fibrinogen-Bestimmungsmethoden zu entnehmen. Mit einer Präzision pro Serie von $2,81 \%$ und einer Präzision von Tag zu Tag mit 3,31\% zeigt die neue Methode eine gute Reproduzierbarkeit.

Beim Erarbeiten dieser neuen, mechanisierten, immunologisch-nephelometrischen Fibrinogen-Bestimmungsmethode wurde darauf geachtet, nicht eine Methode zu präsentieren, die auf Grund von Bestimmungen aus Normalplasmen zu offensichtlich ,richtigen" FibrinogenKonzentrationen kommt. Vielmehr sollte sie vor allem unter extremen Bedingungen geprüft sein. Dazu gehörte das Testen unter Fibrinogenolyse (Abb. 2). Wie die Abbildung zeigt, nimmt unter Plasmin-Aktivierung pro

Tab. 1. Präzision der Fibrinogen-Bestimmungsmethoden mit Standard-Human-Plasma (Batch No. 1174):

\begin{tabular}{|c|c|c|c|c|}
\hline & $\mathbf{N}$ & $\begin{array}{l}\text { Konzentration der } \\
\text { Präzisionskontrolle }\end{array}$ & $\begin{array}{l}\text { VK } \\
{[\%]}\end{array}$ & \\
\hline $\begin{array}{l}\text { Methode nach Clauss } \\
\text { Partigenplatte } \\
\text { neue Methode } \\
\text { neue Methode }\end{array}$ & $\left.\begin{array}{l}10 \\
34 \\
40 \\
10\end{array}\right\}$ & $\begin{array}{rr}1,38 & \mathrm{~g} / 1 \\
1,22 & \mathrm{~g} / 1 \\
11,15 \mathrm{mg} / 1\end{array}$ & $\begin{array}{l}5,16 \\
3,43 \\
2,81 \\
3,31\end{array}$ & $\begin{array}{l}+ \\
+ \\
+ \\
++\end{array}$ \\
\hline
\end{tabular}

$+=$ pro Serie

++ von Tag $2 u$ Tag

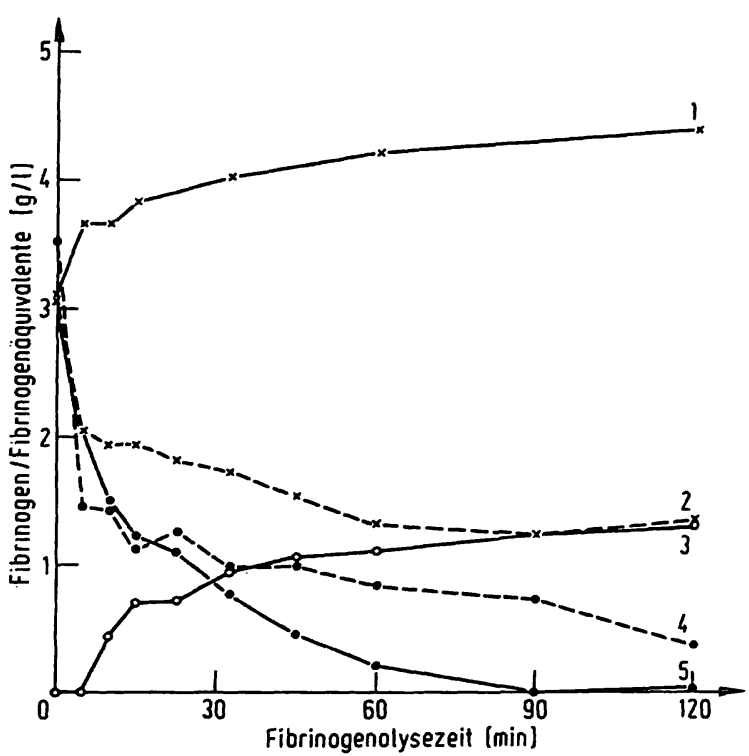

Abb. 2. Bestimmungen von Fibrinogenäquivalenten unter Fibrinogenolyse in Abhängigkeit von der Zeit im Methodenvergleich:

Inkubationsansätze bei $37^{\circ} \mathrm{C}$ :

$\mathrm{Zu} \mathrm{je} \quad 500 \mu \mathrm{l}$ Citratmischplasma $+500 \mu 1$ Test-Ṣtreptokinase ( $\hat{=} 1000$ I.E.)

Zugabe von

$500 \mu \mathrm{l}$ Trasylol ( $\hat{=} 10000 \mathrm{KIE}) \mathrm{zu}$ den jeweiligen

Ansätzen nach $0,5,10,15,22,5,32,5,45,60,90$ und

120 Minuten Inkubationszeit.

$1=$ einfache radiale Immundiffusion auf $M$-Partigenplatte,

4 = Schnell-Fibrinogengerinnungs - Methode nach Clauss.

Neue, mechanisierte, immunologisch-nephelometrische

Schnellmethode:

$\mathbf{2}=$ Plasma-Fibrinogenäquivalente $(=, a$ " $)$,

$\mathbf{3}=$ Serum-Fibrinogenäquivalente (= „Spaltprodukte") $\left(=, b^{\prime \prime}\right)$,

$5=$ Differenzkurve $=2-3=$ gerinnbares Fibrinogen. 
Zeiteinheit die gerinnbare Fibrinogen-Konzentration dieses Mischplasmas sehr schnell ab und ist nach 120 Minuten mit der neuen, mechanisierten Methode nicht mehr nachweisbar. Zum Zeitpunkt 0 Minuten bestand eine Konzentration von 3,09 g/l und war nephelometrisch nach 120 Minuten unter die Nachweisgrenze abgesunken. Gleichzeitig ist aus der Abbildung das entsprechende Ansteigen der nicht gerinnbaren Fibrinogen-

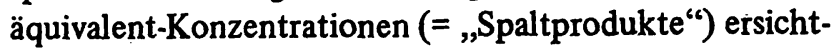
lich, die gleichfalls mit der neuen, mechanisierten Methode ermittelt wurden. Zum Zeitpunkt 0 Minuten noch nicht nachweisbar, stiegen sie nach 120 Minuten auf $1,32 \mathrm{~g} / 1$ an. Mit dem gleichen fibrinogenolytischen Probenmaterial wurden ebenfalls Fibrinogen-Bestimmungen mit der einfachen radialen Immundiffusion auf der Partigenplatte und mit der Methode nach Clauss durchgeführt. Wie Abbildung 2 deutlich zeigt, mißt die Partigenplatten-Methode unter Fibrinogenolyse im Gegensatz zur Methode nach Clauss und der neuen, mechanisierten, immunologisch-nephelometrischen Methode völlig divergierende Wertee. Denn trotz ähnlicher Antiserum-Qualität aller hier durchgeführten immunologischen Methoden ergab die auf dem Prinzip der radialen Immundiffusion basierende Methode bei fibrinogenolytischem Probenmaterial scheinbar höhere „Fibrinogen“-Werte. Offensichtlich kann die Partigenplatten-Methode nicht zwischen Fibrinogen und Fibrinogen-Spaltprodukten differenzieren, so daß längere Diffusionsstrecken der niedermolekulareren Fibrinogenolyse-Produkte höhere „Fibrinogen“-Werte vortäuschen. In dem Maße, in dem etwa die "Spaltprodukte“ zunehmen, nimmt die „Fibrinogen“-Konzentration bei der Partigenplatte zu. Zum Zeitpunkt 0 Minuten beträgt die Konzentration 3,13 g/l und nach 120 Minuten $4,41 \mathrm{~g} / 1$. Ebenfalls mit dem fibrinogenolytischen Material durchgeführte zweidimensionale Geldoppeldiffusions-Teste nach Ouchterlony zeigten, wie zu erwarten war, Mehrbandigkeit in der Fibrinogenolyse-Zeit ab 10 bis 60 Minuten. Ganz im Gegensatz dazu und wider Erwarten zeigten die Partigenplatten-Versuche jeweils nur einfache Präzipitationsringe. Im Gegensatz zu der hier vorgestellten neuen immunologisch-nephelometrischen Fibrinogen-Bestimmungsmethode kann die Partigenplatten-Methode nach dem herkömmlichen Vorgehen sowieso nicht zwischen gerinnbarem Fibrinogen und anderen nicht gerinnbaren Fibrinogenäquivalenten unterscheiden. $\mathrm{Zu}$ erwarten waren von vornherein auf der Partigenplatte unkorrekte Werte aus fibrinogenolytischem Probenmaterial, da bei dem Vorgehen alle immonologisch-faßbaren Fibrinogenäquivalente mitreagieren. Andererseits aber ist die PartigenplattenMethode zu unempfindlich, um ein ähnliches Vorgehen wie bei der Nephelometrie mit getrennten Plasma- und Serum-Proben zu realisieren. So mißt sie aus Material unter Fibrinogenolyse-Bedingungen weder Fibrinogen, noch Fibrinogen-,,Spaltprodukte“, noch Plasma-Fibrinogenäquivalente korrekt. Dies ist bedingt durch unterschiedliche Molekülgrößen von Fibrinogen und Fibri-
nogenolyse-Produkten mit ungleichen Diffusionsstrecken und deren gegenseitiger positiver und negativer Beeinflussung bezüglich Diffusion. Wie aus Abbildung 2 ersichtlich, nehmen die immunologisch-nephelometrisch bestimmten Plasma-Fibrinogenäquivalente unter Fibrinogenolyse ab. Hinzu kommt, daß die Partigenplatte erst nach Tagen ablesbar und deshalb gleichfalls für eine akute Diagnostik unbrauchbar ist.

Entsprechend kritische Einwände wurden schon 1971 von Brittin et al. (6) bei der Bewertung der einfachen radialen Immundiffusion zur Ërmittlung von korrekten Fibrinogen-Werten aus fibrinogenolytischem Patientenmaterial erhoben.

Ëbenso können immunologisch-nephelometrische Methoden ohne differenziertes Vorgehen beim Bestimmen von Fibrinogenäquivalenten nicht den Anspruch auf „Richtigkeit" erheben, sofern es sich um spaltprodukt-haltiges Material handelt $(2,7,8)$.

Die Abbildung 2 zeiğt zudem die „Fibrinogen"-Werte nach der Clauss'schen Methode aus dem fibrinogenolytischen Probenmaterial. Zum Zeitpunkt 0 Minuten beträgt die Fibrinogen-Konzentration 3,52 g/l und nach 120 Minuten nur noch $0,38 \mathrm{~g} / \mathrm{l}$. In der ,Frühphase“ der Plasminolyse laufen die Werte der Clauss'schen Methode recht gut übereinstimmend mit dem nephelometrisch ermittelten gerinnbaren Fibrinogen. In der ,Spätphase“ der Plasminolyse jedoch mißt die Clauss'sche Methode im Vergleich zur Nephelometrie höhere „Fibrinogen“Werte. Wie die Abbildung zeigt, ergeben sich nach der Clauss'schen Methode in der späteren Phase Mittelwerte, die etwa zwischen den nephelometrisch ermittelten gerinnbaren Fibrinogen-Werten und den Vollplasma-Fibrinogenäquivalenten liegen. Unsere Befunde über höhere gemessene „Fibrinogen“-Werte in der Fibrinogenolyse"Spätphase“" mit der Clauss'schen Methode im Gegensatz zur neuen, immunologisch-nephelometrischen $\mathrm{Me}$ thode könnten damit erklärt werden, daß thrombingerinnbare Fibrinogen-Derivate, vor allem aber die Spaltprodukte X (9), hier erfaßt wurden. Auf Grund des bis zu 85-fach konzentrierteren Proben-Ansatzes für die Methode nach Clauss geht besonders dieser Fehler bei niedrigen Fibrinogen-Konzentrationen, sprich wenig vorverdünnten Proben, ein.

Da bekannt ist, daß Fibrinogen-Werte durch hohe Heparin-Konzentrationen im Plasma falsch-niedrig bei der Methode nach Clauss ausfallen können, wurden ebenfalls mit den drei zur Diskussion stehenden Methoden Fibrinogen-Bestimmungen mit steigenden Heparin-Zusätzen durchgeführt (Abb. 3). Wie deutlich zu erkennen ist, sind beide immunologischen Methoden über den ausgetesteten Bereich von fast 40 USP-Einheiten Heparin pro ml Vollplasma unbeeinflußt. Im Gegensatz dazu kommt es bei der Clauss'schen Bestimmungsmethode ab 10 USPEinheiten Heparin pro ml Vollplasma (ausgehend von einer 1:10-Vorverdünnung des Citratplasmas) zu scheinbar verminderten Fibrinogen-Konzentrationen infolge der 


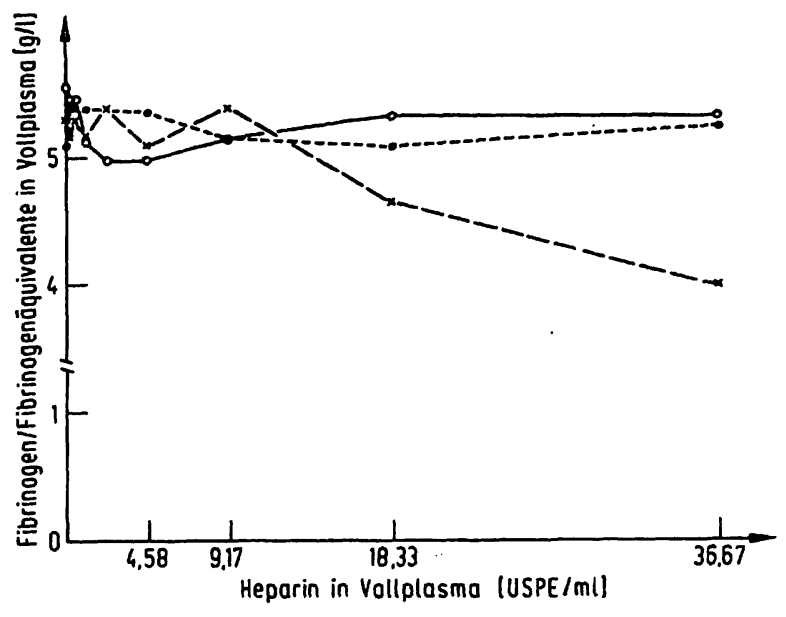

Abb. 3. „Fibrinogen“-Bestimmungen im Methodenvergleich bei verschiedenen Heparin-Konzentrationen: Ansätze: $\mathrm{Zu}$ je $500 \mu l$ Citratmischplasma $+500 \mu \mathrm{l}$ Trasylol $(\triangleq 10000 \mathrm{KIE})$ wurden verschieden konzentrierte Heparin-Lösungen in $100 \mu \mathrm{l}$-Pipettierungen dazugegeben. Es resultierten Heparin-Konzentrationen von 0,0 bis 36,67 USP-Einheiten pro ml Vollplasma.

0 Partigenplattenmethode

- - neue Methode

$\mathrm{x}---\mathrm{x}$ Methode nach Clauss.

Thrombin-Inhibierung. Obwohl klinisch auch diese Heparin-Konzentrationen praktisch nicht vorkommen, sind falsche Laborwerte denkbar. Im Reagenzglas-Versuch sind höhere Heparin-Konzentrationen, die das Meßergebnis nach der Clauss'schen Methode verfälschen können, durchaus möglich, wenn heparin-haltiges Probenmaterial bei niedrigen Fibrinogen-Konzentrationen sehr viel konzentrierter (z. B. 1:2) eingesetzt werden muß oder die Abnahme-Spritze heparin-kontaminiert war.

Zum Uberprüfen der neuen Methode gehörte auch das Untersuchen von Patientenmaterial. Dazu wurde aus 180 Citratblutproben gemischten Krankenguts gleichfalls im Methodenvergleich mit der einfachen radialen Immundiffusion auf der Partigenplatte und der Methode nach Clauss das gerinnbare Fibrinogen mechanisiert, immunologisch-nephelometrisch bestimmt. Tabelle 2 gibt die Ergebnisse der Methodenvergleiche untereinander mit Korrelationskoeffizienten und bivariaten Regressionsgeraden wieder. Aus Tabelle 3 sind die Methoden-Mittelwerte mit

Tab. 2. Fibrinogen-Methodenvergleiche mit Korrelationskoeffizient $(r)$ und bivariater Regressionsgerade $(y=a x \pm b)$ : $N=180$ Patientenproben gemischten Krankenguts:

\begin{tabular}{llll}
\hline & r & \multicolumn{2}{c}{$y=a x \pm b$} \\
& & a & b \\
\hline A / Clauss & 0,831 & 0,978 & $+0,064$ \\
A / Partigenplatte & 0,812 & 0,932 & $+0,759$ \\
Partigenplatte / Claussis & 0,770 & 1,050 & $-0,733$ \\
B / Partigenplatte & 0,864 & 0,978 & $+4,778$ \\
B / Clauss & 0,874 & 1,027 & $-0,518$ \\
\hline
\end{tabular}

neue Methode: $\mathbf{A}=$ gerinnbares Fibrinogen B = Plasma-Fibrinogenäquivalente
Tab. 3. Methodenmittelwerte ( $\bar{x})$, Standardabweichungen ( $\pm \mathrm{s}$ ) und Mittelwertsabweichung in \% vom nephelometrisch ermittelten gerinnbaren Fibrinogen $(=100 \%)$ aus $180 \mathrm{~Pa}$ tientenproben gemischten Krankengutes:

\begin{tabular}{llll}
\hline & $\begin{array}{l}\overline{\mathbf{x}} \\
{[\mathrm{g} / \mathrm{l}]}\end{array}$ & $\begin{array}{l} \pm \mathrm{s} \\
{[\mathrm{g} / \mathrm{l}]}\end{array}$ & $\begin{array}{l}\text { Mittelwertsabweichung } \\
{[\%] \text { von A }}\end{array}$ \\
\hline Clauss & 2,943 & 1,268 & $-0,02$ \\
Partigenplatte & 3,500 & 1,208 & $+18,92$ \\
$\mathrm{~B}$ & 3,370 & 1,235 & $+14,51$ \\
$\mathrm{~A}$ & 2,943 & 1,297 & \\
\hline
\end{tabular}

neue Methode: $\mathbf{A}=$ gerinnbares Fibrinogen

B $=$ Plasma-Fibrinogenäquivalente

Standardabweichung der 180 Patienten-Fibrinogen-Werte und zusätzlicher Angabe der prozentualen MittelwertsAbweichung vom Mittelwert des nephelometrischermittelten, gerinnbaren Fibrinogen ersichtlich.

Tabelle 4 gibt die Signifikanz-Berechnungen, die aus den jeweiligen Methoden-Mittelwerten und deren Standardabweichung nach dem t-Test errechnet wurden, wieder.

Die graphischen Darstellungen (Abb. 4 und 5) geben die ermittelten Einzelwerte des nephelometrisch-bestimmten, gerinnbaren Fibrinogens in Gegenüberstellung zu Partigenplatten-Werten (Abb. 4) und denen der Clauss'schen Methode (Abb. 5) wieder.

$\mathrm{Zu}$ den Fibrinogen-Bestimmungen aus 180 Patientenproben kann durch das statistische Auswerten der Versuchsergebnisse eine ähnliche, wenn auch nicht ganz so krasse, Schlußfolgerung wie bei den beschriebenen Fibrinogenolyse-Versuchen gezogen werden.

Für die Partigenplatte ergab sich auch hier ein statistisch hoch signifikanter, höherer Mittelwert gegenüber den Werten nach der Clauss'schen und der nephelometrischen Methode. Dies ist eindeutig durch das Miteingehen von fibrinogen-äquivalenten „Spaltprodukten“ bedingt.

Die Werte nach der Clauss'schen und der nephelometrischen Methode ergaben im Mittel gleiche Ergebnisse. Im Einklang mit den Befunden aus dem Fibrinogenolyse- und dem Heparin-Versuch heben sich bei der Clauss'schen Methode hier offensichtlich im Mittel die,

Tab. 4. Signifikanz-Berechnungen (p) nach dem $t-T$ est mit $\bar{x}$ und $s$ der benutzten Fibrinogen- bzw. FibrinogenäquivalentBestimmungs-Methoden aus 180 Patientenproben gemischten Krankenguts:

\begin{tabular}{lll}
\hline & p & \\
\hline A / Clauss & $>0,995$ & nicht signifikant \\
A / Partigenplatte & $<0,001$ & hoch signifikant \\
Partigenplatte / Clauss & $<0,001$ & hoch signifikant \\
B / Partigenplatte & $>0,30$ & nicht signifikant \\
B / Clauss & $<0,005$ & signifikant \\
A / B & $<0,005$ & signifikant \\
\hline
\end{tabular}

neue Methode: $\mathbf{A}=$ gerinnbares Fibrinogen

B = Plasma-Fibrinogenäquivalente 


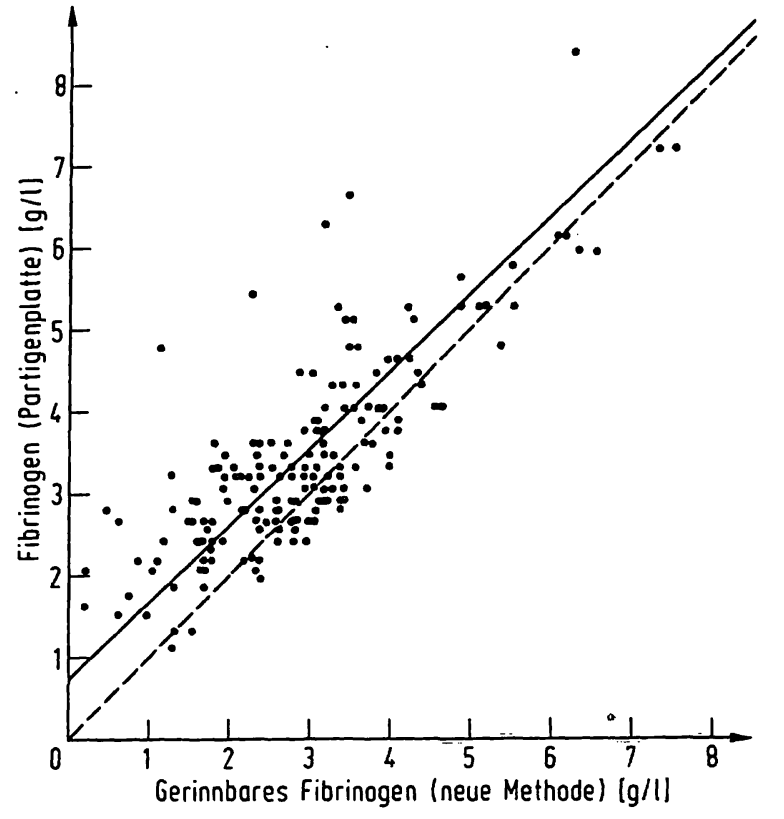

Abb. 4. Fibrinogen-Methodenvergleich mit 180 Patientenproben gemischten Krankengutes: -

_ bivariate Regressionsgerade $--x=y$.

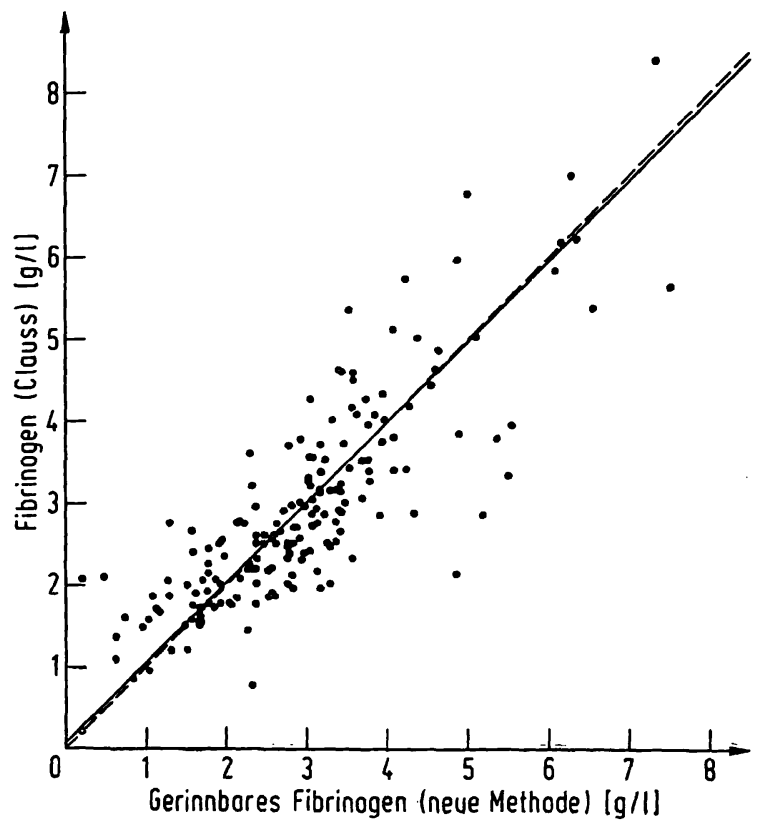

Abb. 5. Fibrinogen-Methodenvergleich mit 180 Patientenproben gemischten Krankengutes:

_ bivariate Regressionsgerade nach unserer Meinung, falsch-höheren „Fibrinogen“Werte der „Spätphase“-Fibrinogenolyse gegen die falschniedrigen unter Heparin-Einfluß wieder gegeneinander auf.

Schon 1964 forderte Groß (10) eine zuverlässige und schnelle Fibrinogen-Bestimmungsmethode für die Klinik. Gefragt sind dabei Methoden, die sowohl das gerinnungsaktive wie auch das immunologisch-erfaßbare Fibrinogen korrekt und plausibel bestimmen. Diese Forderung ist bis heute auch in der einschlägigen Literatur immer noch aktuell. So stellen auch Stevens et al. (11) 1973 und Barthels (12) 1975 Diskrepanzen bei ihren durchgefuihrten Methoden-Vergleichen fest und kommen zu dem unbefriedigenden Ergebnis, daß nur unter „bestimmten “ Voraussetzungen eine „bestimmte“ Methode in „,bestimmten“ Fällen vielleicht zu ,richtigen“ FibrinogenWerten kommt.

Hier muß nochmals festgestellt werden, daß sich aus unseren Normalplasmen-Untersuchungen praktisch keine Fibrinogen-Wertedifferenżen bei den untersuchten drei Methoden zeigten. Aus diesem Grund sind in dieser Arbeit zur Beurteilung der zitierten Fibrinogen-Bestimmungsmethoden bewußt Maßstäbe angelegt worden, wie sie bei Patienten herrschen oder herrschen könnten.

Abșchließend kann gesagt werden, daß fibrinogenolyțisches Probenmaterial sogar tendentiell falsch von der Partigenplatten-Methode bestimmt wird. Ganz im Gegensátz dazu erhält man mịt der Schnellmethode nach Clauss plausible Werte in der fibrinogenolytischen ,Frühphase“. In der „Spätphase“ liegen die Werte zu hoch, wenn auch tendentiell richtig. Jedoch kommt es bei der Clauss'schen Methode, im Gegensatz zu den immunologischen Bestimmungen, żu falsch-niedrigen Fibrinogen-Werten bei Heparin-Konzentrationen ab 10 USP-Einheiten Heparin/ml Vollplasma. Die neue immunologisch-nephelometrische Schnellmethode unterscheidet und mißt quantitativ gerinnbare und nicht gerinnbare Fibrinogenäquivalente, wobei unter gerinnbaren Fibrinogenäquivalenten nur das gerinnbare Fibrinogen zu verstehen ist. Die neue Methode mißt korrekte und tendentiell plausible Fibrinogen-Werte, auch unter Fibrinogenolyse und Heparin-Einfluß.

\section{Danksagung:}

Fräulein Sabine Krone danke ich für sorgfältiges technisches Mitarbeiten. 


\section{Literatur}

1. Clauss, A. (1957), Acta Haematol. 17, 237-246.

2. Ebeling, H. (1973), diese Z. 11, 209-214.

3. Ebeling, H. (1974), diese Z. 12, 54-58.

4. Ebeling, H. (1974), diese Z. 12, 423-426.

5. Störiko, K. (1968), Blut 16, 200-208.

6. Brittin, G. M., Rafina, H., Raval, D., Werner, M. \& Brown, B. (1972), Amer. J. Clin. Path. 57, 89-94.

7. Dessert, J., Bocquet, P., Belleville, J., Later, R. \& Quincy, C. (1973), Haemostasis 1, 215-222.

8. Linzana, J., Jansson, L. \& Hellsing, K. (1975), Clin. Chem. $21,762-765$.
9. Marder, V. J. \& Budzynski, A. Z. (1975), Thrombos. Diathes. Haemorrh. 33, 199-207.

10. Groß, R. (1964), Behringwerk-Mitteilungen, 44, S. 76,

Thrombolyse-Therapie mit Streptokinase, Bericht über das II. Streptase-Kolloquium, Bad Nauheim, Januar 1964, N. G. Elwert, Universitäts- und Verlags-Buchhandlung, MarburgLahn.

11. Stevens, D. J. \& Sanfelippo, M. J. (1973), Amer. J. Clin. Path. 60, 182-187.

12. Barthels, M. (1975), Ärztl. Lab. 21, 295-302.

Dr. H. Ebeling

Institut fü Klinische Chemie und Klinische Biochemie am

Klinikum Steglitz der

Freien Universität Berlin

Hindenburgdamm 45

D-1000 Berlin 45 
